\title{
LES LOIS DE L'HÉRÉDITÉ
}

\author{
Par M. LICIEN CLENOT
}

Correspondant de l'Institut,

Professeur à la Faculté des Sciences de Nancy.

Pendant longtemps, les connaissances sur l'Hérédité ont consisté en une collection de faits décousus, soment contradictoires et médiocrement observés, dont on ne pouvait déduire ancune règrle ; la transmission des caractères héritables semblail pleine de caprices et d'incertitudes. Ce n'est qu'à partir de i goo que l'on a commencé à voir clair ; quelques botanistes et zoologistes, travaillant indépendamment les uns des autres, découvrirent à peu près en mème temps ou plutôt retrouvèrent les premières lois simples de l'hérédité, qui avaient été formulées avant eux, vers I 865 ı 869 , par un moine autrichien, Johann Mwries. (Gregor de son nom de religieux).

Depuis 19oo, une pléiade de chercheurs ont considérablement étendu nos acquisitions sur ce sujet, qui est maintenant connu dans les grandes lignes d'une façon définitivis. Mais, pour bien comprendre les règles de l'hérédité, de façon à pouvoir les utiliser dans le domaine de la pratique, il faut faire un effort pour s initicr; malheureusement la plupart des éleveurs reculent devant la technicité des termes biologiques et renoncent à s'assimiler des connaissances qui leur sont cependant indispensables pour les guider avec certitude dans leurs essais de croisement et de sélection. C'est dans l'espoir de faciliter cette initiation que j ai écrit le présent article (I).

Presque toutes les espèces de plantes cultivées ou d'animaux domestiques (que nous comnaissons nalurellement beaucoup micux que les plantes et animaux sauvages) présentent ce qu'on appelle des races ou variétés; les traits descriptifs de celles-ci, tels que la couleur du pelage, la taille, la longueur des poils, etc., sont des caracteres de ces races. Or, il est évident que ces caractères sont contenus en puissance dans les cellules microscopiques, l'œuf et le spermalozoïde, qui par leur union ont fondé l'individu : dans l'ocuf d'où sorlira un Lapin angora, par exemple, il y a quelque chose qui est responsable du caractère argora ; nous appellerons ce quelque chose facteur du caractère angora. Disons tout de suite qu'il paraît prouvé que le facteur est une particule matérielle, d'une extraordinaire petitessc, qui est logée dans le noyau cellulaire, et qu'on doit admettre que le noyau de l'ocuf, comme celui du spermatozö̈de, renferme

(I) Le lecteur ciésireux d'approfondir le sujet pourra lire l'un des owrages suivants : - Cú́xot, La Genèse des Espèces animales, $2^{\mathrm{e}}$ édil., Paris, Alcan, т 92 т ; - Grvéxot, L'Hérédilé (Encyclopédie scientifique, Doin, Paris, I924) ; - Jean Rosand, Les chromosomes, artisans de l'hérédité el du sexe, Hachet.te, Paris, $19^{2} 8$. 
un nombre considérable de ces particules qui déterminent les divers caractères de la race considérée : l'ensemble des particules constitue le patrimoine héréditaire de la race ; quand un éleveur achète une monte, c'est-à-dire les spermatozoïdes d'un étalon de valeur, il achète, en réalité, un patrimoine héréditaire apprécié.

Chaque futur individu, au moment de sa naissance, c'est-à-dire au moment de la fécondation de l'ouf, se trouve donc avoir un patrimoine héréditaire double, l'un qui vient du parent maternel (noyau de l'œuf), l'autre qui vient du parent paternel (noyau du spermatozoïde). Lorsque les deux patrimoines sont rigoureusement identiques, l'individu est dit de race pure; il est commode d'employer un mot technique, synonyme de race pure, qui est le mot homozygole (ce terme, tiré du grec, vient de zygote qui est l'œuf fécondé, et de homoios, semblable, qui fait allusion à l'identité des deux patrimoines). Quand les deux patrimoines ne sont pas tout à fait identiques (pour 1,2 ou $n$ facteurs), l'individu est de race impure ou hétérozygote (dı grec hetercs, différent). Par exemple, si on accouple un Lapin gris, à poil ras (de race pure), avec une Lapine blanche, à pelage angora (également de race pure), on obtiendra des produits hétérozygotes, que l'on appelle aussi hybrides, qui seront deux fois hétérozygotes, une fois pour la couleur et une fois pour la longueur du poil.

Lorsqu'on connaît les parents d'un individu donné, il est facile d'affirmer, comme ci-dessus, l'hétérozygotie de celui-ci ; mais, si l'on prend dans un élevage un individı quelconque, dont l'ascendance n'est pas connue, son aspect ne révèle pas avec certitude sa qualité d'homozygote ou d'hétérozygote (sauf dans certains cas où il y a une mosaïque visible de deux couleurs différentes, comme dans une Chatte noire et orange). Ainsi le Lapin hybride de l'exemple donné plus haut, qui est sûrement hétérozygote pour deux facteurs, est en tout semblable extérieurement à son père de racc pure (nous verrons pourquoi tout à l'heure) : comme lui, il est gris à poil ras, et ne présente aucun indice du blanc et du poil angora apportés potentiellement par le patrimoine maternel. Lorsqu'on acquiert un reproducteur qui répond parfaitement à la définition d'une race donnée, on ne peut pas savoir, avant de l'avoir cssayé, si c'est un animal homozygote vraiment de race pure, ou bien si c'est un hétérozygote qui a sculement l'apparence extérieure de la race pure. A vrai dire, cela est tout à fait indifférent á beaucoup d'éleveurs marchands, qui n'ont en vue que la vente de leurs produits, et qui affirment froidement (peut-être de bonne foi ia pureté raciale d'un individu sur son simple aspect. Lorsqu'on fait des expériences de laboratoire sur l'hérédité, on cherche toujours à prendre comme point de départ un animal sauvage, qui a toutes les chances d'ètre homozygote : si l'on n'a point cette ressource, il suffit de croiser entre eux deux individus dont on désire éprouver l'homozygotic, puis de recroiser leurs descendanits entre eux ou avec les parents pendant quelques générations (inbreeding) : si toutes ces générations sont parfaitement identiques entre elles et avec les parents 
originels, sans aucune apparition de caractères nouveaux, on pourra être assuré de la parfaite homozvgotie de la lignée, qui est une race pure ou lignée pure.

On est arrivé à définir les trois lois fondamentales de l'hérédité en faisant des expériences très simples, les plus simples possible. Une fois bien comprises, le reste n'est plus que cas particulier.

\section{$1^{\text {re }}$ loi : Duminance ou serri-dominance}

Commençons par étudier ce qui se passe lorsquion croise deux individus qui diflèrent par un caractère nettement oppositif, par exemple la présence ou l'absence de coulcur. Ivant l'époque mendélienne, on crovait volontiers qu'à la suite d'un tel croisement il y avait dans le produit une sorte de mélange, comme lorsqu'on additionne de l'eau et du vin, ou une mosaïque, comme dans une lleur panachée : ce n'est pas tout à fait cela. Deux cas se présentent :

$I^{\circ}$ Croisement d'une Souris colorée par une Souris albinos, c'est-àdire ne possédant pas le facteur de la couleur. Les petits sont tous, infailliblement, identiques au farent coloré, sans aucun affaiblissement de la teinte ; on dit alors qu'il y a dominance du facteur couleur sur le facteur absence de couleur, lequel est dit dominé.

$2^{\circ}$ Croiscment d'une Belle-de-Nuit (Mirabilis jalapa) à fleurs rouges par un Mirabilis à fleurs blanches ; les pieds hybrides portent tous des fleurs rose foncé, plus claires que celles du iype rouge. La teinte rouge de l'un des parents a donc été affaiblie par le blane de l'autre : on dit alors qu'il y a mélange ou dominance incomplète.

La première loi de l'héréditć est donc celle de la dominance ou de la semi-dominance d'un caraclère sur son symétrique.

\section{$2^{\circ}$ loi : Disjenction des facleurs chez les hybrides}

D'après ce que nous avons dit plus haut, les hybrides résultant des croisements pris ci-dessus comme exemples ont un patrimoine héréditaire double : celui qui renferme le facteur de couleur est dominant sur celui qui correspond à la coloration blanche, mais ce dernier n'en existe pas moins. Croisons, maintenant, entre cux, deux de ces hétérozygotes de première génération (que l'on appelle $F_{I}$ pour abréger); ils vont donner une deuxième génération (ou $\mathrm{F}$ ?), dont nous ne pouvons comprendre la constitution que si nous connaissons une deuxième loi de l'hérédité.

Lorsque les hétérozygotes de la $F_{\text {I }}$ ont formé leurs aufs ou leurs spermatozoïdes, il s'est passé un phénomène tout à fait particulier que l'on appelle la disjonction des factcurs. Dans les cellules génitales, les deux potentialités que possédaient les hétérozygotes se séparent, comme si elles ne pouvaient plus désormais cohabiter dans la même cellule : la moitié des cufs ou des spermatozoïdes reçoit le facteur couleur, mais celui-là 
seulement ; tandis que I'autre moitié reçoit le facteur du blanc, mais celuilà seulement. Chaque ouf prît à la fécondation ou chaque spermatozoïde n'a donc plus qu'un facteur en simple dose. Le croisement de deux hétérozygotes est, en réalité, la fécondation de deux sortes d'oufs en nombre égal par deux sortes de spermatozoïdes également en nombre égal, de sorte que toutes les combinaisons pọssibles peuvent se réaliser, dans des proportions qui ressortent clairement lersqu'on a opéré sur des nombres suffisamment grands. C'est exactement comme si l'on jetait en l'air deux pièces de monnaie : tantôt on aurait 2 côtés pile, tantôt 2 còtés face, ou bien un pile et une face, et ceci dans un rapport numérique parfaitement

Fig. 1. - Diagramme du croisement mendélien type entre une Souris à pelage colcré et une Souris albinos (les symboles employés pour dés:gner los facteurs sont ici différents de ce: $x$ cin texte: $\Lambda$ désigne le facteur de l'absence de couleur ; la fraction $\mathrm{C} / \mathrm{I}$ indique que $\mathrm{C}$ est $\mathrm{do-}$ minant sur $A$. Le cercle noir qui recouvre partiellement un centre blanc est également le symbole de la dominance du facteur $C$ s:r le facteis $A$.

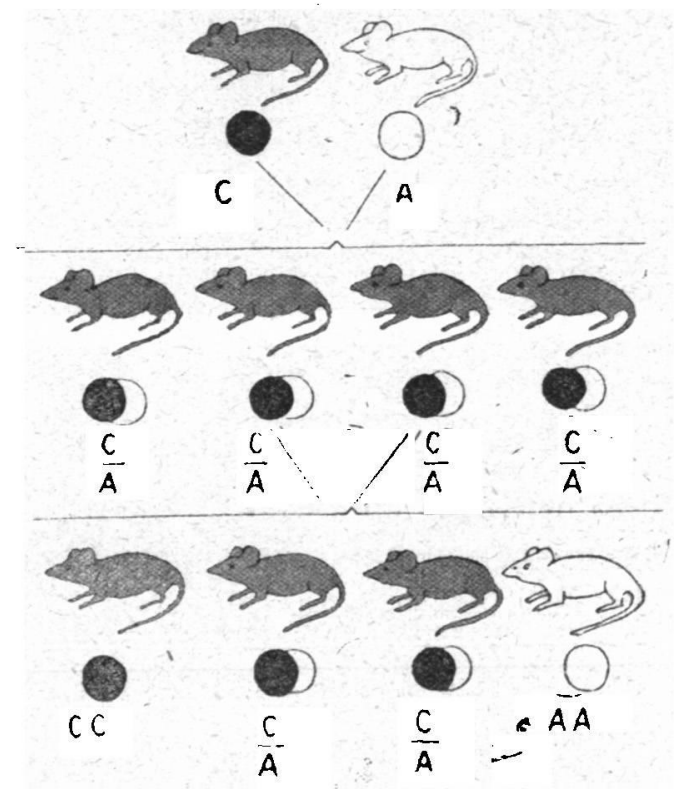

constant, pourvu que l'on fasse un nombre suffisant de jels : ce rapport serait de $n$ face, $n$ pile et $2 n$ face et pile.

Revenons à l exemple concret $n^{\circ}$ I (celui des Souris); appelons $C$ le facleur (dominant) de la couleur, et $c$ celui (dominé) de l'albinisme : les cufs en nombre égal $C$ et $c$ ont, respectivement, autant de chances les uns que les autres d'être fécondés par les spermatozoïdes en nombre égal $C$ et $c$ : on a donc / combinaisons possibies, $C C, c c, C c, c C$. La première est un homozygote coloré, exactement comme le grand-parent homozygote $C C$ et comme les parents hétérozygotes de la $F_{I}$; la deuxième est un homozygote albinos, exactement semblable au grand-parent $c c$; quant aux deux combinaisons $C c$ et $c L$, en raison de la dominance de $C$ sur $c$ ( $\mathrm{I}^{\mathrm{ri}}$ loi), elles donnent des Souris colorées identiques, comme aspect et constitution patrimoniale, aux hétérozygotes de la $F_{I}$, et comme aspect à l'homozygote CC. (Figure 1.) 
Nous pouvons écrire ces croisements sous une forme diagrammatique, qui permet de tout envisager d'un coup d'ocil :

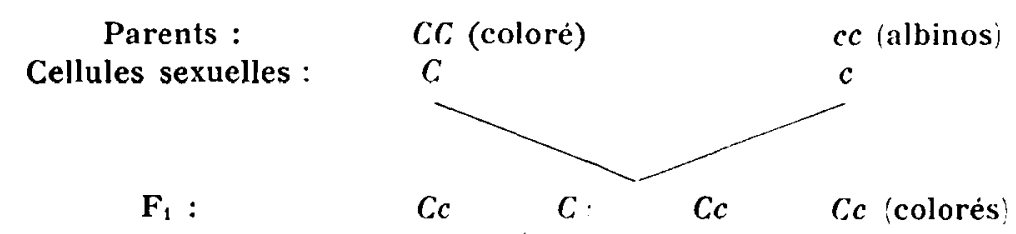

Cellules sexuel'es après disjonction

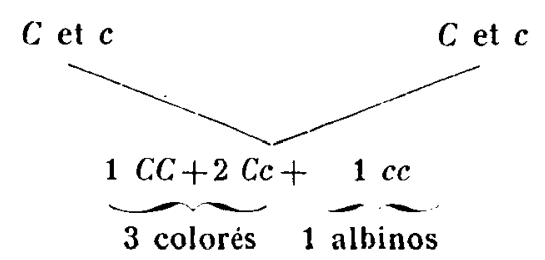

Cetle proportion de 3 et I est la proportion mendélienne typique, dans le cas de la dominance complète d'un facteur sur son symétrique.

Dans l'exemple concret $n^{\circ}$ ? (Belle-de-Nuit), mème résultat, à cela près qu'en vertu de la règle de semi-dominance, on distingrue facilement, à l'œil, l'homozygote $C$ C (lleurs rougres) et les 2 hétérozygrotes Cc (rose foncé) : la $F_{2}$, tout à fait comme dans le jeu des pièces de monnaie, se compose visiblement de I $C C$ (rouge), 2 Ce (rose foncé) et I cc (blanc).

La deuxième loi est donc celle de la disjonction des facteurs et de leur recombinaison libre; on l'appelle la disjonction mendélienne, en l'honneur de Mradel qui, le premier, l'a reconnue avec précision.

\section{$3^{\text {e }}$ loi : Independance des facteurs.}

Nous allons réaliser, maintenant, une expérience un peu plus compliquée, en croisant deux individus qui diffèrent l'un de l'autre, non pas par un unique caractère oppositif, mais par deux caractères ; par exemple une Souris blanche valseuse ( $\mathrm{I}$ ) avec une Souris colorée ì marche normale. Dans la première génération hybride ( $\left.\mathrm{I}_{\mathrm{l}}\right)$ ), la loi de dominance complète joue : toutes les Souris sont identiques au parent coloré à marche normale : il y a en effet dominance du facteur couleur sur l'absence de couleur (ce que nous savons déjà) et dominance du facteur de la marche rectiligne sur la valse.

La seconde génération ( $\left.\mathbf{F}_{2}\right)$, produite par le croisement de deux hỵbrides de la Fr, est complexe d'aspect ; elle comprend 4 sortes de Souris : deux sont identiques aux grands-parents (Souris colorée à marche rectiligne, Souris albinos valseuse), et deux sont nouvelles (Souris colorée valseuse, Souris albinos à marche rectiligne). Iorsqu'on a opéré sur des nombres

(r) La Souris valseuse, que l'on peut se procurer facilement chez les marchands de petits animaux, a la curieuse propricté de ne pouvoir progresser qu'en décrivant des courbes, et de se tourner parfois sur elle-mème avec une extrême rapidité. La Souris valseuse japonaise est ćlevée depuis très longtemps en Exlrìme-Orient. 
suffisamment grands, on constate que les nombres respectifs de ces 4 sortes de Souris sont entre eux comme les nombres 9-3-3-1 (9 Souris colorées à marche rectiligne, 3 Souris colorées valseuses, 3 abinos à marche rectiligne, I albinos valseusə). A vant toute explication, nous pouvons déjà remarquer la symétrie mendélienne de ces chiffres, où nous retrouvons deux fois la proportion fatidique 3-i : il y a en effet i s Souris colorées $(9+3)$ contre 4 blanches $(3+1)$, et il y a aussi I 2 Souris à marche rectiligne contre 4 valseuses. (haque facteur se comporte donc comme s'il était seul, en se disjoignant de son partenaire ; c'est la loi de l'indépendance des facteurs.

Le résultat global 9-3-3-r peut être expliqué de la façon la plus claire : appelons $R$ et $r$ les facteurs dominant et dominé correspondant à la marche rectiligne et à la valse, et $C$ et $c$ les facteurs dominant et dominé correspondant à la couleur et à l'albinisme. Les hybrides de $F_{I}$ résultent donc de l'addition des deux patrimoines héréditaires $C R$ et $c r, C$ et $R$ dominant $c$ et $r$, ce qui donne des Souris colorées à marche rectiligne. Lorsque ces hybrides forment leurs cellules sexuelles, il y a disjonction indépendante des facteurs symétriques $\left(2^{\circ}\right.$ Joi), sous la condition expresse que l'cruf ou Je spermatozoïde reçoive un patrimoine renfermant les deux sortes de facteurs : il y a donc des ceufs de formule $C R,(i r, c R, c r$, en nombres égaux, et de même des spermatozoïdes de formule $C R, C r, c R, c r$, toujours en nombres égaux. La fécondation des 4 sortes d'cufs par les 4 sortes de spermatozoïdes, avec des chances égales, donne donc if combinaisons possibles, que l'on peut calculer commodément avec le tahleau ci-dessous. La première ligne horizontale représente les \& sortes d'ceufs possibles, et la première ligne verticale les $\{$ sortes de spermatozoïdes possibles ; dans les cases se trouvent les i6 combinaisons possibles, qui, en fait, se réalisent rigoureusement.

\begin{tabular}{|c|c|c|c|c|}
\hline & $C R$ & $c R$ & $C r$ & $c r$ \\
\hline$C R$ & $C R \cap R$ & CeRR & $C R r$ & $C c \dot{R} r$ \\
\hline$c R$ & $\operatorname{CcR} R$ & $c c R R$ & $C c R r$ & $c c R r$ \\
\hline$c r$ & $C C R r$ & $C c R r$ & $\cos r$ & Ccrr \\
\hline$c r$ & $C c R r$ & $c c R r$ & Ccr. & $c c r r$ \\
\hline
\end{tabular}

On voit dans ce tableau qu'il y a 9 combinaisons, de formules variées, renfermant les deux facteurs dominants $C$ et $R$ : ce sont les 9 Souris colorées à marche rectiligne, dont une seule est homozygote et identique au grand-parent dominant CCRR, toutes les autres étant hétérozygotes pour I ou 2 facteurs ; -3 combinaisons renferment le facteur dominant $C$ et le 
facteur dominé $r$, ce dernier en double dose ; ce sont des colorées valseuses dont une seule $\mathrm{CCr}$ est homozygote : -3 combinaisóns renferment le facteur dominé $c$ cn`double dose et le facteur dominant $R$; ce sont des albinos à marche rectiligna, dont une seule ccRR est homozygote. Enfin une seule combinaison, identique au grand-parent dominé, n'a que les facteurs deminés $c$ et $r$; c'est une Souris albinos valseuse ecrr, homozygote. Sur 16 Souris, il n'y en a donc que 4 qui soient homozygotes; le reste est hétérozygute pour $\mathrm{I}$ ou 2 facteurs.

On peut essayer une à une les Souris produites et constater que leur formule factorielle est bien celle que leur assigne la théorie.

Ciette loi de l'indépendance des facteurs et de leur disjonction propre a une importance capitale; elle nous permet de comprendre comment l'Homme a pu agir si profondément sur les animaux domestiques : mais en dépit des apparences, il n'a rien créé de vraiment nouveau; il a réuni dans des races homoúygotes (ce que l'on appelle des races bien fixées) ce qui était épars chez des individus divers. Chez un Bovidé, par exemple, il a pu se proposer de réaliser la combinaison suivante : les cornes courtes, une certaine couleur du pelage, une certaine précocité de développement, des facultés laitières ou beurrières supérieures : si les facteurs correspondants existent (mais épars) dans son troupeau, la réussite de-Ia combinaison désirée est certaine : c'est affaire de temps et de patience, et aussi de compréhension des règles de l'hérédité.

Bien entendu, on peut continuer la vérification de l'indépendance des facteurs, en croisant des animaux qui diffèrent par $3,4, \ldots n$ caractères oppositifs ; les résultats deviennent de plus en plus complexes ; par exemple, pour 3 caructères oppositifs, on a en $F_{2}$ les proportions de 27-9-9-9-3-3-3-1. Par le calcul, on peut prévoir exactement la constitution et les nombres relatifs des produits du croisement des. hybrides les plus compliqués.

\section{Correctifs : Relations entre caracières et facteurs}

Dans les expériences fondamentales rapportées plus haut, nous nous sommes exprimés, dans un but nécessaire de clarté, comme si les caractères dont on éturliait l'hé rédité étnient déterminés chacun par un facteur particulier. Or, la relation entre caractère et facteur n'est pas si simple que cela.

Nous pouvons considérer les facteurs comme des substances chimiques spéciales, renfermées en grand nombre dans les cellules sexuelles, héritables indépendamment par lo mécanisme de la disjonction, qui déterminent, à travers les innombrables divisions et réactions cellulaires, une certaine constitution intime des cellules de la peau, du système nerveux, du tube digestif, etc. ; quand un facteur est modifiè (ce qu'on appelle une mutation), il en résulte un: autre constitution des cellules du corps entier, qui peut se traduire par des caractères visibles, élablissant une différence avec le type nor mutć. La variation visible et mesurable que nous percevons dans une race ou une variété n'est que le symptôme d'un 
changement intime, qui, peut-être, porte également, mais d'une façon invisible, sur bien d'autres parties. Les différentes modifications, visibles ou invisibles, liées au changement d'un facteur unique, sont dites corrélatives : c'est ainsi que le Chat à yeux bleus est sourd, qu'une Souris valseuse est sourde, que le (hien ì peau nue a des malformations dentaires, etc. ; une Souris ou un Rat albinos, dont un certain facteur $C$ a été changé en un facteur $c$, ne présente aucune couleur pigmentaire dans la peau, non plus que dans les yeux, et son psychisme par rapport à celui de la Souris sauvage colorée est profondément modifié, puisque l'animal devient apprivoisable. Il n'est donc pas tout à fait exact de dire (comme nous l'avons fait de propos délibéré dans les pages ci-dessus) que $C$ est le facteur de couleur et sa mutation $c$ celui de l'albinisme, que $R$ est le facteur de la marche rectiligne ot sa mutation $r$ celui de la valse ; $c$ est aussi bien le facleur du caractère apprivoisable et $r$ celui de la surdité.

Ce n'est pas tout : un caractère que l'on peut désigner d'un seul mot, pelage noir par exemple, est bien en rapport avec un facteur spécial, mais sa réalisation, la seule chose qui nous importe au fond, dépend plus ou moins de l'interaction d'autres facteurs : ainsi, on sait qu'il existe un facteur, que nous appellerons $p$, qui conditionne la panachure, c'est-àdirc la présence de zones blanches tranchant sur un fond coloré ; $p$ est une mutation du facteur $P$ qui correspond à un pelage de teinte uniforme, sans zones blanches. Or, il est évident que pour qu'il y ait panachure visible, il faut qu'il y ait un fond de couleur ; en fait, un albinos peut posséder le facteur $p$, mais celui-ci reste complètement inopérant, puisque la robe est parfaitement blanche par suite de la présence du facteur $c$. La panachure dépend donc, non seulement de $p$, le facteur spécial, mais aussi de $C$, le facteur de couleur. Bien entendu, l'albinos, dans un croisement, pourra transmettre le facteur $p$, c'est-à-dire qu'il transmettra un caractère qui n'était pas exprimé chç lui. De même un Taureau peut transmettre des facteurs de bonne ou manvaise laitière, un Coq des facteurs de bonne ou mauvaise pondeuse, qui ne peuvent naturellement pas s'exprimer dans le sexe mâle.

Il faut bien comprendre qu'en hérédité, il faul ponser focteurs et non pas caractères ; le caractere visible, sur lequel nous sommes bien obligés de nous guider, n'est que le symptôme de l'evistence d'un facteur, et sa réalisation peut dépendre d'autres facteurs ou mème de conditions extérieures. Ce qui se transmet, c'est le facteur, aussi bien celui qui se traduit par un caractère visıble que celui qui est latent ou dissimulé dans le patrimoine, soit parce qu’il est dominé, soit parce qu'il n'a pu s'exprimer.

Voilà, exposé aussi simplement que possible, l'essentiel des règles de l'Hérédité, applicables à toutes les plantes et à tous les animaux. Dans un autre article, nous traiterons des ras particuliers qui permettront, avec ces lois, de résoudre les problèmes de la pratique. 